\title{
Determination of the polyphenol and antioxidant activity of different types and forms of millet
}

\author{
A. Almaski, S. Thondre, H. Lightowler and S. Coe \\ Functional Food Centre, Department of Sport and Health Sciences, Oxford Brookes University, Gipsy Lane, \\ Headington, Oxford.
}

The demand for functional foods has recently increased, partly due to the growing evidence of several grains and their positive effect on health ${ }^{(1)}$. Millet is one of such functional grains known for significant health benefits that are attributed to its high content of polyphenols, antioxidants and dietary fibre ${ }^{(2)}$. Foods such as millet may exert their effect by improving insulin sensitivity and reducing the glycaemic response due in part to a high antioxidant and polyphenol content. Therefore, millet consumption may help to prevent or reduce the risk of developing Type 2 diabetes.

The aim of this study was to determine and compare the antioxidant activity and polyphenol content of different types of millet grains (pearl millet, finger millet, foxtail millet, common millet, little millet, barnyard millet, kodo millet) in different forms (seeds, flour and flakes).

The total polyphenol content of the millet products was measured using the Folin-Ciocalteu method (FCR) ${ }^{(3)}$. Antioxidant activity was measured using the ferric ion-reducing antioxidant power (FRAP) method $^{(4)}$.

Table 1. Polyphenol content (mg GAE/g) and total antioxidant activity (mmol/g) of each type and form of millet (mean \pm SD)

\begin{tabular}{|c|c|c|c|c|c|}
\hline Millet Type & Test & Grain & Flour & Flakes & P-value \\
\hline \multirow[t]{2}{*}{ Pearl millet } & Polyphenol & $2 \cdot 15 \pm 0 \cdot 22$ & $1.59 \pm 0.23$ & $1.76 \pm 0.06$ & $<0.001$ \\
\hline & FRAP & $4 \cdot 58 \pm 0 \cdot 64$ & $4.313 \pm 0.322$ & $4.737 \pm 0.32$ & 0.028 \\
\hline \multirow[t]{2}{*}{ Finger millet } & Polyphenol & $3 \cdot 72 \pm 0 \cdot 14$ & $2 \cdot 55 \pm 0 \cdot 20$ & $1.27 \pm 0.14$ & $<0.001$ \\
\hline & FRAP & $13.98 \pm 0 \cdot 16$ & $9 \cdot 87 \pm 1.02$ & $11.46 \pm 1 \cdot 34$ & $<0.001$ \\
\hline \multirow[t]{2}{*}{ Little millet } & Polyphenol & $1.51 \pm 0.21$ & $0.52 \pm 0.06$ & $0.75 \pm 0.03$ & $<0.001$ \\
\hline & FRAP & $3 \cdot 5 \pm 0 \cdot 26$ & $0.61 \pm 0 \cdot 21$ & $1.34 \pm 0.06$ & $<0.001$ \\
\hline \multirow[t]{2}{*}{ Barnyard millet } & Polyphenol & $1 \cdot 41 \pm 0 \cdot 11$ & $0.51 \pm 0.03$ & $0.69 \pm 0.03$ & $<0.001$ \\
\hline & FRAP & $2 \cdot 69 \pm 0 \cdot 37$ & $0.55 \pm 0 \cdot 37$ & $1 \cdot 010 \pm 0 \cdot 08$ & $<0.001$ \\
\hline \multirow[t]{2}{*}{ Foxtail millet } & Polyphenol & $0.98 \pm 0 \cdot 06$ & $0 \cdot 29 \pm 0 \cdot 02$ & $0.63 \pm 0.05$ & $<0.001$ \\
\hline & FRAP & $1.89 \pm 0.14$ & $0 \cdot 35 \pm 0 \cdot 05$ & $1 \cdot 12 \pm 0 \cdot 15$ & $<0.001$ \\
\hline \multirow[t]{2}{*}{ Kodo millet } & Polyphenol & $4 \cdot 44 \pm 0 \cdot 15$ & $0 \cdot 22 \pm 0 \cdot 04$ & N/A & $<0.001$ \\
\hline & FRAP & $20 \cdot 81 \pm 1 \cdot 31$ & $0.33 \pm 0 \cdot 13$ & N/A & $<0.001$ \\
\hline \multirow[t]{2}{*}{ Proso millet } & Polyphenol & $1 \cdot 14 \pm 0 \cdot 11$ & $0.54 \pm 0.04$ & N/A & $<0.001$ \\
\hline & FRAP & $4.35 \pm 0 \cdot 62$ & $0 \cdot 92 \pm 0 \cdot 11$ & N/A & $<0.001$ \\
\hline
\end{tabular}

Data was tested for normality using the Shapiro-Wilks test. Values are the mean of three independent experiments. The one-way between group ANOVA, independent sample t test, Kruskal Wallis or Mann Whitney test were used to compare between the same millet and different millet forms; $\mathrm{p}<0.05$.

These results show that millet grain is richer in polyphenol and antioxidant content compared to millet flour and flakes. Table 1 shows that kodo millet grain has a higher polyphenol and antioxidant content followed by finger millet in both grain and flour forms. This study represents a starting point for the future human studies that will look at the effect of polyphenol-rich millet products on glycaemic and insulinaemic response.

1. Devi P., Vijayabharathi R., Sathyabama S., Malleshi N., Priyadarisini V. (2014) J Food Sci Technol. 51(6), pp. 1021-1040.

2. Shahidi F., and Chandrasekara A. (2013) J Funct Foods. 5(2), pp. 570-581.

3. Coe S., Fraser A. and Ryan L. (2013) Int J Food Sci. 2013, p.6.

4. Thondre P. S., Ryan L. and Henry C. (2011) Food Chem. 126(1), pp. 72-77. 